\title{
Long non-coding RNA TCF7 predicts the progression and facilitates the growth and metastasis of colorectal cancer
}

\author{
FU-SHU JIN ${ }^{1}$, HONG-MEI WANG ${ }^{2}$ and XIAO-YAN SONG ${ }^{3}$ \\ ${ }^{1}$ Anorectal Section; Departments of ${ }^{2}$ Obstetrics and Gynecology, and ${ }^{3}$ Emergency, \\ Yantai City Hospital of Traditional Chinese Medicine, Yantai, Shandong 264000, P.R. China
}

Received June 8, 2017; Accepted November 30, 2017

DOI: $10.3892 / \mathrm{mmr} .2018 .8708$

\begin{abstract}
Long non-coding RNA (lnc)TCF7 has been reported to promote the self-renewal of human cancer stem cells, and enhance the aggressiveness of human non-small cell lung cancer and hepatocellular carcinoma cells. However, the effect of 1ncTCF7 on colorectal cancer (CRC) tumorigenesis and progression is currently unclear. In the present study, reverse transcription-quantitative polymerase chain reaction results demonstrated that lncTCF7 expression was higher in CRC tissues compared with adjacent normal tissues and was significantly associated with tumor size, differentiation degree, tumor-node-metastasis grade, lymph node metastasis and invasion depth. In addition, 1ncTCF7 demonstrated a high sensitivity and specificity for diagnosing $\mathrm{CRC}$, as determined by receiver operating characteristic curve analysis. Furthermore, lncTCF7 silencing in SW-620 and HT29 CRC cell lines inhibited the proliferation, cell cycle, migration and invasion of cells, as determined by Cell Counting Kit-8 assays, propidium iodide (PI) staining and flow cytometry, wound healing assays and Transwell invasion assays, respectively; however, Annexin V/PI double staining and flow cytometry indicated that lncTCF7 silencing did not significantly affect the apoptosis of CRC cells. These results indicate that lncTCF7 may predict the progression, and promote the growth and metastasis, of CRC, and may therefore be a novel diagnostic marker and therapeutic target for CRC treatment.
\end{abstract}

\section{Introduction}

Colorectal cancer (CRC), a commonly diagnosed cancer, was associated with a high incidence and mortality rates worldwide in 2012. In addition, at present, its incidence and

Correspondence to: Professor Xiao-Yan Song, Department of Emergency, Yantai City Hospital of Traditional Chinese Medicine, 39 Xingfu Road, Zhifu, Yantai, Shandong 264000, P.R. China E-mail: ganzhu85792@163.com

Key words: colorectal cancer, long non-coding RNA TCF7, metastasis, diagnostic marker, therapeutic target mortality rates are increasing (1). In China, CRC was the fifth most common cancer type, with high incidence and mortality rates in 2011 (2). Currently, treatment of patients with relapsed and metastatic CRC is challenging (3). Therefore, there is an urgent requirement for novel early diagnostic markers and therapeutic targets for CRC treatment.

Accumulating evidence has demonstrated that abnormally expressed long non-coding RNAs (lncRNAs) in cancer may regulate the proliferation, apoptosis, metastasis and differentiation of cancer cells by regulating epigenetic, splicing, transcriptional or post-transcriptional processes and subsequently tumorigenesis and tumor progression (4). In renal cell carcinoma, lncRNA (lnc)FTX and lncTUG1 promote cell proliferation, while lncGAS-5 promotes cell apoptosis and inhibits cell proliferation. In addition, certain lncRNAs, including HOTAIR, MALAT1 and linc00152, perform multiple functions, including roles in the promotion of cell proliferation, invasion, migration and metastasis (5). In hepatocellular carcinoma, IncRNAs DANCR, Lalr1 and UFC1 are reported to regulate Wnt signaling to promote or inhibit cancer cell invasion, migration and metastasis, and lncRNAs UCA1 and lncSOX4 were demonstrated to promote cancer cell proliferation (6). In melanoma, IncRNAs Lime23, BANCR, ANRIL and UCA1 regulate cancer cell proliferation and growth, and lncRNAs GAS5, PRC2 and PAUPAR modulate cancer cell invasion, migration and metastasis (7). Previous studies have demonstrated that various IncRNAs, including CCAT1, H19, FEZF-1-AS1, MALAT1 and UCA1, enhance the proliferation, invasion and metastasis of CRC cells (8).

LncTCF7 binds to the T-cell factor 7 (TCF7) gene and recruits the Switch/sucrose non-fermentable (SWI/SNF) complex to the TCF7 promoter to upregulate TCF7 expression and activate Wnt signaling (9). TCF7 serves an important role in T-cell development and multipotential hematopoietic cell self-renewal by activating Wnt signaling $(10,11)$. TCF7 expression is reported to be significantly higher in clear cell renal cell carcinoma tissue compared with normal tissue (12), while TCF7 silencing inhibited the survival of prostate cancer cells and inhibited the development of androgen deprivation resistance in prostate cancer $(13,14)$. TCF7 expression was reported to be increased in human osteosarcoma, and microRNA-192 suppressed TCF7 expression to inhibit cell growth and invasion, and induce apoptosis (15). Furthermore, TCF7 promoted tumorigenesis following the ocular transplantation 
of embryonic stem cell-derived retinal progenitor cells (11). These results from previous studies indicate that lncTCF7 may promote the expression levels of oncogenic TCF7 and may therefore be an oncogene. In addition, previous studies have revealed that IncTCF7 promotes the self-renewal of human cancer stem cells and enhances the aggressiveness of human non-small cell lung cancer and hepatocellular carcinoma cells $(9,16,17)$. However, the effect of lncTCF7 on CRC tumorigenesis and progression remains unclear. In the current study, lncTCF7 expression in CRC tissues was investigated, and the association between lncTCF7 expression and different clinicopathological features of patients with CRC was determined. Furthermore, the effect of lncTCF7 silencing on the proliferation, cell cycle, apoptosis, migration and invasion of CRC cells was investigated. The results demonstrated that lncTCF7 predicted the progression, and promoted the growth and metastasis, of CRC, indicating that lncTCF7 may be a novel diagnostic marker and a potential therapeutic target for CRC.

\section{Materials and methods}

Patients and tissue samples. In total, 112 patients with CRC were recruited from Yantai City Hospital of Traditional Chinese Medicine (Yantai, China) between March 2014 and December 2016. The sex and age distribution of patients is presented in Table I. The CRC patients age ranged from 31-72 years with a median of 56 years. None of the recruited patients with CRC received chemotherapy or radiation therapy prior to surgery. Diagnosis of CRC and clinicopathological characteristics of the patients were confirmed by two pathologists. All participants signed informed consent forms and experiments were approved by the Ethics Committee of the Yantai City Hospital of Traditional Chinese Medicine. CRC tissues and adjacent normal tissues (at least $2 \mathrm{~cm}$ away from the primary cancer site) were obtained by performing radical resection and were immediately stored at $-80^{\circ} \mathrm{C}$ until use for total RNA extraction.

$R N A$ extraction and reverse transcription-quantitative polymerase chain reaction $(R T-q P C R)$. To perform RT-qPCR, total RNA was extracted from CRC tissues and adjacent normal tissues, and CRC cell lines, using TRIzol reagent (Invitrogen; Thermo Fisher Scientific, Inc., Waltham, MA, USA), according to the manufacturer's protocol. Subsequently, mRNA was reverse transcribed into cDNA at $37^{\circ} \mathrm{C}$ for $15 \mathrm{~min}$ and at $85^{\circ} \mathrm{C}$ for $5 \mathrm{sec}$ using a PrimeScript RT Reagent kit with gDNA Eraser (Takara Biotechnology Co., Ltd., Dalian, China), according to the manufacturer's protocol. LncTCF7 expression was determined by performing qPCR with SYBR Premix Ex Taq (Takara Biotechnology Co., Ltd.) and a 7500 Real-Time PCR system (Applied Biosystems; Thermo Fisher Scientific, Inc.). Thermocycling conditions for qPCR was as follow: Initial denaturation at $95^{\circ} \mathrm{C}$ for $5 \mathrm{~min}$, followed by 40 cycles of denaturation at $95^{\circ} \mathrm{C}$ for $10 \mathrm{sec}$ and combined annealing/extension at $60^{\circ} \mathrm{C}$ for $30 \mathrm{sec}$. Sequences of primers used for performing qPCR were as follows: 1ncTCF7 forward, 5'-AGGAGTCCTTGGACCTGAGC-3' and reverse, 5'-AGTGGCTGGCATATAACCAACA-3'; GAPDH forward, 5'-CCCATCACCATCTTCCAGGAG-3' and reverse, 5'-GTT
GTCATGGATGACCTTGGC-3'. GAPDH was used as an internal control for normalizing lncTCF7 mRNA expression. Relative lncTCF7 expression was expressed as fold induction by using the $2^{-\Delta \Delta \mathrm{Cq}}$ method (18). All samples were assessed in triplicate.

Cell culture and small interfering (si)RNA transfection. SW-620 and HT29 CRC cell lines (American Type Culture Collection, Manassas, VA, USA) were cultured in RPMI-1640 (Gibco; Thermo Fisher Scientific, Inc.) supplemented with $10 \%$ fetal bovine serum (Gibco; Thermo Fisher Scientific, Inc.), $100 \mathrm{U} / \mathrm{ml}$ penicillin and $100 \mu \mathrm{g} / \mathrm{ml}$ streptomycin at $37^{\circ} \mathrm{C}$ in a humidified atmosphere of $5 \% \mathrm{CO}_{2}$. LncTCF7 siRNA (si-lncTCF7-1, 5'-AGCCAACATTGT TGGTTA T-3'; and si-lncTCF7-2, 5'-CACCTAGGTGCTCACTGA A-3') and negative control siRNA (si-NC, 5'-UUCUCCGAA CGUGUCACGUTT-3') were purchased from Guangzhou RiboBio Co., Ltd., (Guangzhou, China). SW-620 and HT29 cells $\left(3 \times 10^{5}\right)$ were transfected with the siRNAs $(50 \mathrm{nM})$ using Lipofectamine 2000 (Invitrogen; Thermo Fisher Scientific, Inc.), according to the manufacturer's protocol. A total of $48 \mathrm{~h}$ following transfection, lncTCF7 expression was determined by performing RT-qPCR.

Proliferation assay. A cell proliferation assay was performed using Cell Counting Kit (CCK)-8 assay kit reagent (Beyotime Institute of Biotechnology, Haimen, China), according to the manufacturer's protocol. At $24 \mathrm{~h}$ following transfection, Cells in si-negative control (NC) and si-lncTCF7 groups were trypsinized and cultured in 96-well plates in triplicate at a density of $5 \times 10^{3}$ cells per well at $37^{\circ} \mathrm{C}$ in a humidified atmosphere of $5 \% \mathrm{CO}_{2}$. Following culture for $0,24,48$ and $72 \mathrm{~h}, 10 \mu \mathrm{l}$ CCK-8 reagent was added to each well and the cells were incubated for a further $4 \mathrm{~h}$ at $37^{\circ} \mathrm{C}$. Absorbance was measured at a wavelength of $450 \mathrm{~nm}$ using a microplate reader.

Apoptosis and cell cycle assay. Cell apoptosis assay was performed using the Annexin V-FITC apoptosis detection kit (Nanjing KeyGen Biotech Co., Ltd., Nanjing, China). For investigating apoptosis, SW-620 and HT29 cells were digested with trypsin and were centrifuged at $200 \mathrm{x} \mathrm{g}$ for $5 \mathrm{~min}$ at $4^{\circ} \mathrm{C}$ $48 \mathrm{~h}$ after transfection. Subsequently, cell pellets were resuspended in $500 \mu \mathrm{l}$ binding buffer, which was followed by the addition of $5 \mu \mathrm{l}$ Annexin $\mathrm{V}$-fluorescein isothiocyanate and $5 \mu \mathrm{l}$ propidium iodide (PI) to the cell suspension, and the cells were incubated in the dark for $15 \mathrm{~min}$ at room temperature. Cell apoptosis was detected by performing flow cytometry using a BD FACSCalibur system and data was analyzed using the CellQuest software version 5.1 (BD Biosciences, Franklin Lakes, NJ, USA). For the cell cycle assay, SW-620 and HT29 cells were fixed in $500 \mu 170 \%$ pre-cooled ethanol at $4^{\circ} \mathrm{C}$ overnight. An equal amount of PBS was added twice for washing. A total of $100 \mu \mathrm{l}$ RNase A was added at $37^{\circ} \mathrm{C}$ for $30 \mathrm{~min}$, followed by the addition of $100 \mu \mathrm{l} \mathrm{PI}$ at $4^{\circ} \mathrm{C}$ in the dark for $30 \mathrm{~min}$. Cell cycle analysis was performed by flow cytometry using a BD FACSCalibur system (BD Biosciences) and data was analyzed using the ModFit software version 4.1 (Verity Software House, Inc., Topsham, ME, USA). Each experiment was repeated three times. 
Table I. Association between clinicopathological characteristics and lncRNA TCF7 expression in patients with CRC.

lncRNA TCF7 expression

\begin{tabular}{|c|c|c|c|c|}
\hline Clinicopathological characteristics & All patients, $n=112$ & Low group, $\mathrm{n}=56$ & High group, $\mathrm{n}=56$ & P-value \\
\hline Sex & & & & 0.705 \\
\hline Male & 54 & 26 & 28 & \\
\hline Female & 58 & 30 & 28 & \\
\hline Age, years & & & & 0.674 \\
\hline$<50$ & 40 & 19 & 21 & \\
\hline$\geq 50$ & 72 & 37 & 35 & \\
\hline Location & & & & 0.693 \\
\hline Colon & 70 & 34 & 36 & \\
\hline Rectum & 42 & 22 & 20 & \\
\hline Organization & & & & 0.674 \\
\hline Adenocarcinoma & 48 & 22 & 26 & \\
\hline Mucinous carcinoma & 64 & 34 & 30 & \\
\hline Tumor size, $\mathrm{cm}$ & & & & $0.001^{\mathrm{a}}$ \\
\hline$<5$ & 73 & 45 & 28 & \\
\hline$\geq 5$ & 39 & 11 & 28 & \\
\hline Differentiation & & & & $0.005^{\mathrm{a}}$ \\
\hline Poor & 29 & 8 & 21 & \\
\hline Moderate + high & 83 & 48 & 35 & \\
\hline Lymph node metastasis & & & & $0.010^{\mathrm{a}}$ \\
\hline Yes & 23 & 6 & 17 & \\
\hline No & 89 & 50 & 39 & \\
\hline Depth of invasion & & & & $0.027^{\mathrm{a}}$ \\
\hline Serosa layer & 37 & 13 & 24 & \\
\hline Muscular layer & 75 & 43 & 32 & \\
\hline TNM grade & & & & $0.004^{\mathrm{a}}$ \\
\hline $\mathrm{I}+\mathrm{II}$ & 65 & 40 & 25 & \\
\hline III+IV & 47 & 16 & 31 & \\
\hline
\end{tabular}

${ }^{a} \mathrm{P}<0.05$ between patients with CRC with a high and low lncTCF7 expression. LncTCF7, long non-coding RNA TCF7; CRC, colorectal cancer; TNM, tumor-node-metastasis.

Cell migration and invasion assays. Cell migration was determined using a wound healing assay, according to a method described previously (19). SW-620 and HT29 cells transfected with si-NC and si-lncTCF7 were seeded into 6-well plates $\left(5 \times 10^{5}\right.$ cells/well), and after reaching $90 \%$ confluency, a scratch was made in the plate with a $200 \mu$ l pipette tip. The remaining cells were washed with RPMI-1640 medium and incubated with $5 \% \mathrm{CO}_{2}$ at $37^{\circ} \mathrm{C}$ for $48 \mathrm{~h}$. An image of the migration distance of cells was captured using a FSX100 Biological Image system (Olympus Corporation, Tokyo, Japan) and the images were analyzed using Image $\mathbf{J}$ version 6.0 (Media Cybernetics, Inc., Rockville, MD, USA). For invasion assays, the upper chamber had been precoated with Matrigel (BD Biosciences) and incubated for $24 \mathrm{~h}$. Then cell invasion was evaluated by performing a Transwell assay, according to a method described previously $(20,21)$. Cells in six independent fields per well were imaged at magnification, $\mathrm{x} 200$ by using a microscope (Olympus
Corporation), and the number of migrating and invading cells were counted.

Statistical analysis. Statistical analysis was performed using SPSS version 19.0 software (IBM Corp., Armonk, NY, USA). Continuous variables were presented as the mean \pm standard deviation. Differences between CRC tissues and adjacent normal tissues were analyzed using an independent t-test. Differences among si-NC, si-lncTCF7-1 and si-lncTCF7-2 groups were evaluated using one-way analysis of variance followed by least significant difference post-hoc test. Differences between si-lncTCF7 and si-NC groups were also analyzed using the independent t-test. A receiver operating characteristic (ROC) curve was applied to evaluate the diagnostic value (sensitivity and specificity). According to sensitivity and specificity calculated by SPSS, the value of the Youden index (sensitivity + specificity-1) was calculated, and the best diagnostic cutoff value is the maximum value of the 
A

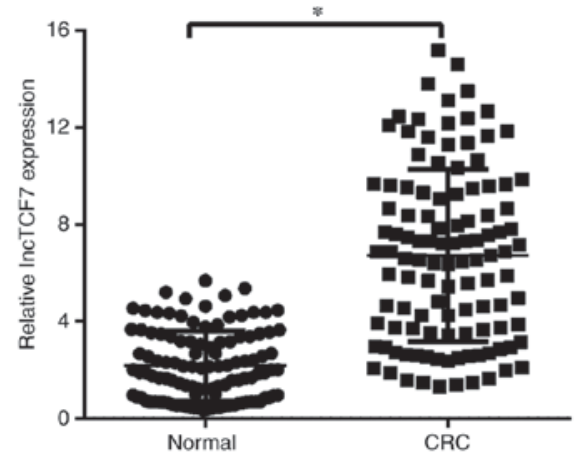

B

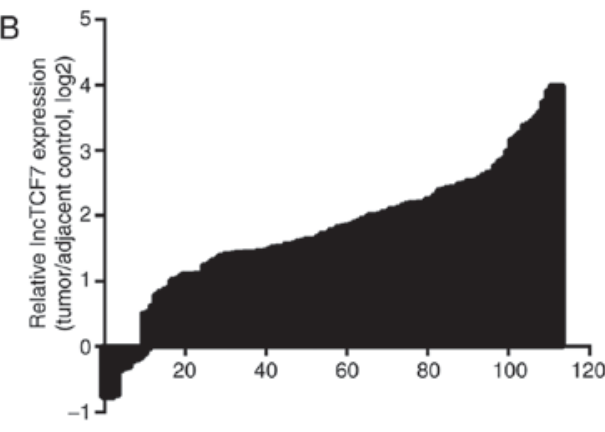

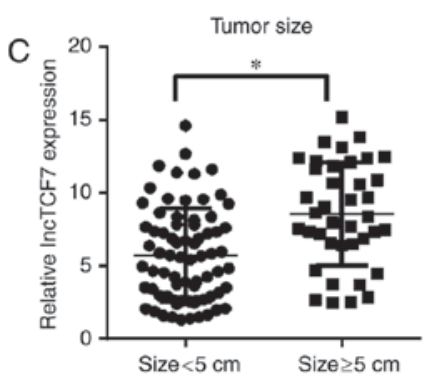
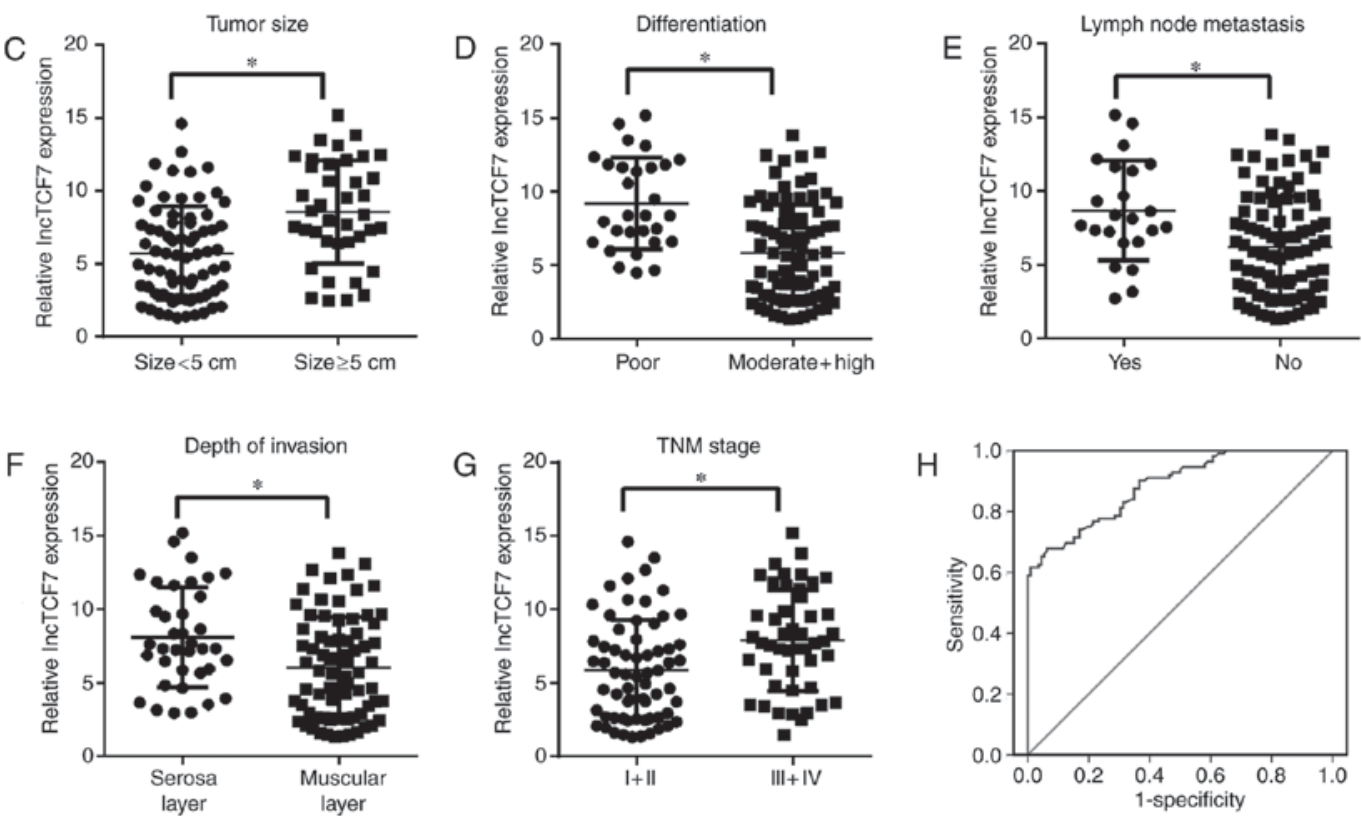

Figure 1. LncTCF7 was overexpressed in CRC tissues and its expression was associated with tumor size, differentiation degree, TNM grade, lymph node metastasis, depth of invasion and CRC diagnosis. (A) LncTCF7 expression in CRC tissues and adjacent normal tissues was evaluated by performing reverse transcription-quantitative polymerase chain reaction. (B) Distribution plot of $\log 2$ values of relative expression ratio (lncTCF7 expression in CRC tissues/lncTCF7 expression in adjacent normal tissues) vs. the number of patients with CRC. LncTCF7 expression in CRC tissues of patients with (C) large- and small-sized tumors, (D) poor and moderate + high tumor differentiation, (E) with or without lymph node metastasis, (F) muscular and serosa layer invasion and (G) TNM grades I + II and III + IV tumors. Data are presented as the mean \pm standard deviation. (H) Receiver operating characteristic curve analysis for assessing the diagnostic value of lncTCF7 expression in CRC. "P<0.05, as indicated. LncTCF7, long non-coding RNA TCF7; CRC, colorectal cancer; TNM, tumor-node-metastasis; lncTCF7, long non-coding RNA TCF7; AUC, area under curve.

Youden index. $\mathrm{P}<0.05$ was considered to indicate a statistically significant difference.

\section{Results}

LncTCF7 expression is increased in CRC tissues. LncTCF7 expression in CRC tissues and adjacent normal tissues was analyzed by performing RT-qPCR. Results of RT-qPCR revealed that lncTCF7 expression (mean value) was significantly increased in CRC tissues compared with adjacent normal tissues $(\mathrm{P}<0.05$; Fig. 1A). In addition, results of RT-qPCR demonstrated that $102(102 / 112 ; 91.07 \%)$ patients with CRC exhibited higher lncTCF7 expression in CRC tissues compared with adjacent normal tissues (Fig. 1B). To investigate the association between the different clinicopathological features of patients with CRC and lncTCF7 expression, the patients were divided into the following two groups based on the comparison between lncTCF7 expression and the median lncTCF7 expression value (6.59) of all CRC tissues: Low expression group (IncTCF7 expression value, <6.59) and high expression group (lncTCF7 expression value, $\geq 6.59$ ). Results for lncTCF7 expression are presented in Table I. LncTCF7 expression was significantly associated with tumor size, differentiation degree, tumor-node-metastasis (TNM) grade, lymph node metastasis and depth of invasion $(\mathrm{P}<0.05)$, but was not significantly associated with sex, age, tumor location or organization $(\mathrm{P}>0.05)$. Furthermore, IncTCF7 expression was increased in patients with large tumors (size, $>5 \mathrm{~cm}$ ) compared with patients with small tumors (size, $<5 \mathrm{~cm}$; Fig. 1C), in patients exhibiting poor tumor differentiation compared with patients exhibiting moderate + high tumor differentiation (Fig. 1D), in patients with lymph node metastasis compared with patients without lymph node metastasis (Fig. 1E), in patients with serosa layer invasion compared with patients with muscular layer invasion (Fig. 1F) and in patients with TNM grade III + IV tumors compared with patients with TNM grade I + II tumors (Fig. 1G). Receiver operating characteristic (ROC) curve analysis was performed to determine the sensitivity and specificity of lncTCF7 for diagnosing CRC based on 

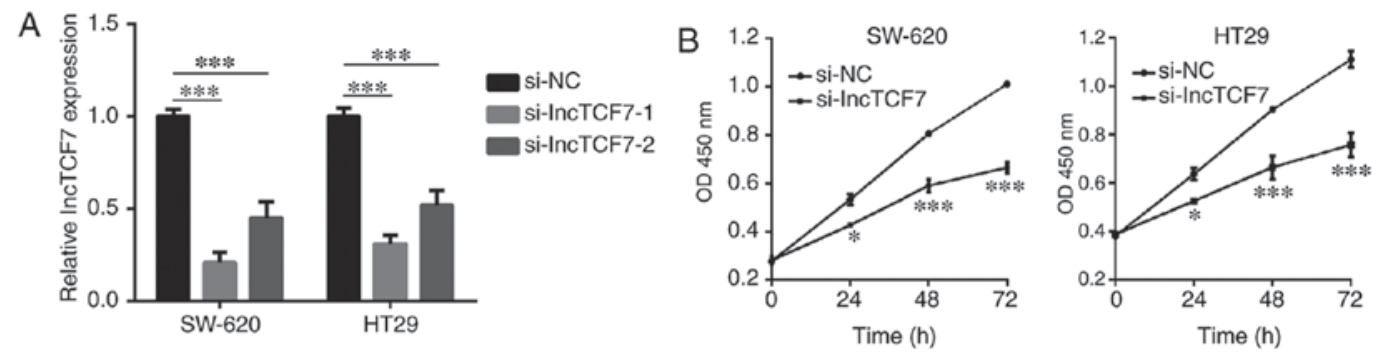

C

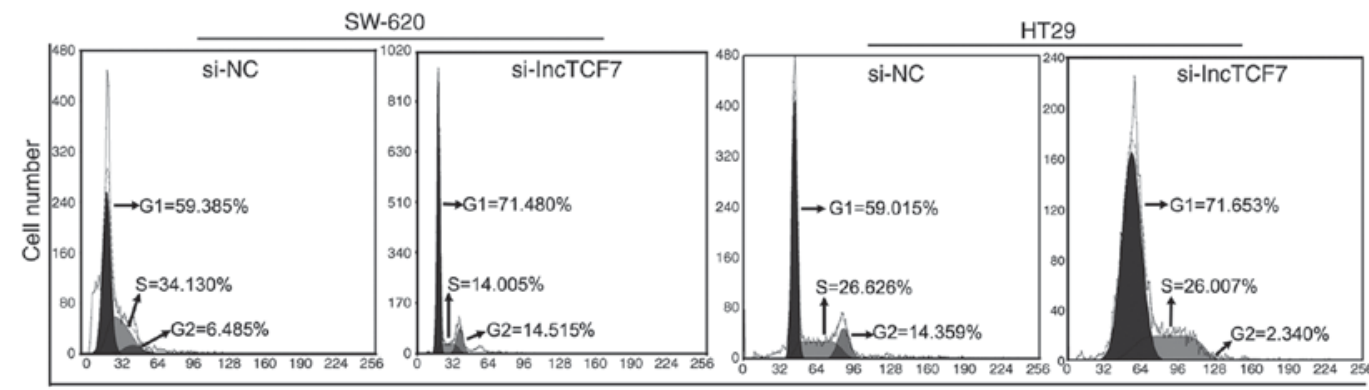

DNA content

D

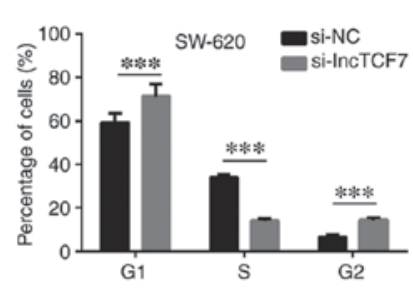

$\mathrm{E}$

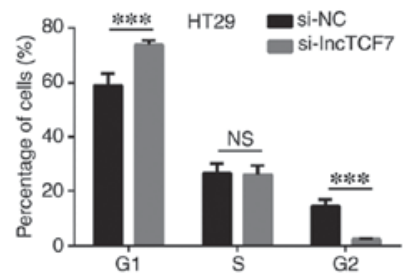

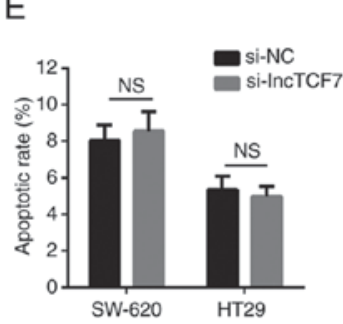
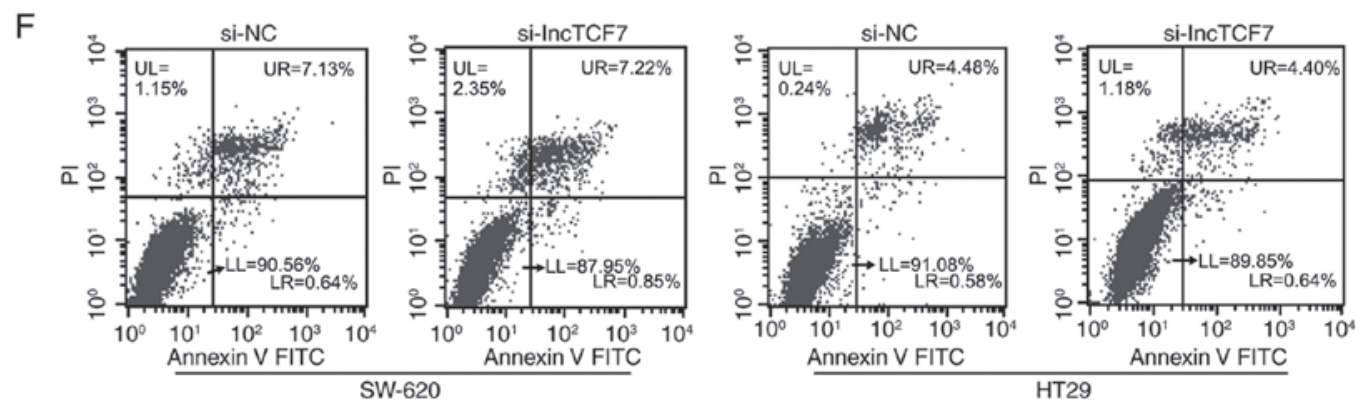

Figure 2. Silencing of lncTCF7 inhibited the proliferation and cell cycle without affecting the apoptosis of SW-620 and HT29 cells. (A) LncTCF7 expression in si-NC- and si-lncTCF7-1/2 transfected SW-620 and HT29 cells was detected by performing reverse transcription-quantitative polymerase chain reaction. (B) Proliferation of si-NC- and si-lncTCF7-transfected SW-620 and HT29 cells was determined using a Cell Counting kit-8 assay. (C) Representative images of the cell cycle of SW-620 and HT29 cells. (D) Cell cycle stages of si-NC- and si-lncTCF7-transfected SW-620 and HT29 cells were investigated and quantified by performing flow cytometry following staining with PI. (E) Apoptosis of si-NC- and si-lncTCF7-transfected SW-620 and HT29 cells was determined by performing flow cytometry following staining with Annexin V-FITC and PI. (F) Representative flow cytometry plots of the apoptosis of SW-620 and HT29 cells. Cells in upper right (UR) + lower right (LR) quadrants were considered as apoptotic cells. Data are presented as the mean \pm standard deviation. For parts A and $\mathrm{D},{ }^{* * * *} \mathrm{P}<0.001$, as indicated; for part $\mathrm{B},{ }^{*} \mathrm{P}<0.05$ and ${ }^{* * * *} \mathrm{P}<0.001$ vs. si-NC group. LncTCF7, long non-coding RNA TCF7; si, small interfering RNA; si-NC, si-negative control; PI, propidium iodide; FITC, fluorescein isothiocyanate; OD, optical density; NS, not significant.

its expression. The ROC curves demonstrated significant separation between the CRC tissues and adjacent normal tissues, with an area under the curve of 0.885 (95\% confidence interval, 0.844-0.926; Fig. 1H). According to sensitivity and specificity calculated by SPSS, the maximum value of the Youden index was 0.616 , and the corresponding optimal lncTCF7 expression cutoff value was 4.48. At the optimal expression cutoff value, the sensitivity and specificity of lncTCF7 for diagnosing CRC were 67.9 and $93.7 \%$, respectively.
LncTCF7 silencing suppresses proliferation without affecting the apoptosis of CRC cells. To evaluate the effect of lncTCF7 expression on the biological function of CRC, SW-620 and HT29 cells were transfected with si-lncTCF7 and lncTCF7 expression was quantified by performing RT-qPCR. Results of RT-qPCR revealed that lncTCF7 expression was significantly inhibited in si-lncTCF7-1- and si-IncTCF7-2-transfected cells, to a larger extent in si-lncTCF7-1-transfected cells, compared with si-NC-transfected cells $(\mathrm{P}<0.05$; Fig. $2 \mathrm{~A})$. These results 
A

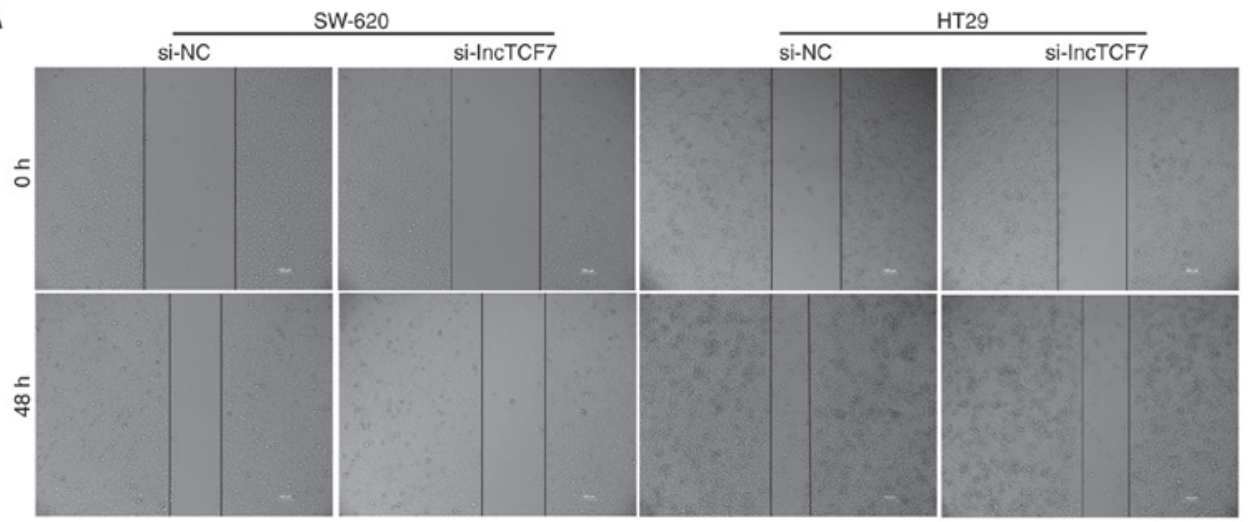

B
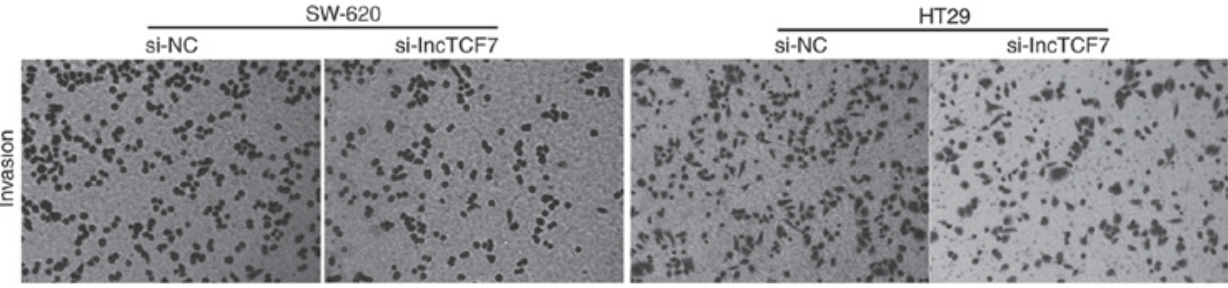

C
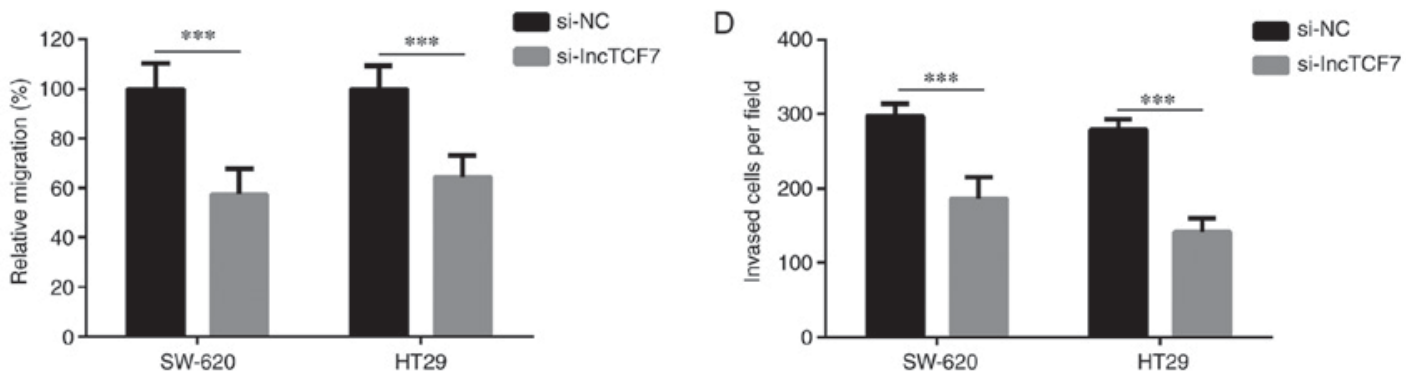

Figure 3. LncTCF7 silencing suppressed the migration and invasion of SW-620 and HT29 cells. (A) Representative images of migrating SW-620 and HT29 cells following wound healing assay (magnification, x100). (B) Representative images of invading SW-620 and HT29 cells (magnification, x200). (C) Relative number of migrating cells per field among si-NC- and si-lncTCF7-transfected SW-620 and HT29 cells. (D) Average number of invading cells per field among si-NC- and si-lncTCF7-transfected SW-620 and HT29 cells. Data are presented as the mean + standard deviation. ${ }^{* * *}$ P $<0.001$, as indicated. LncTCF7, long non-coding RNA TCF7; si, small interfering RNA; si-NC, si-negative control.

indicated that si-lncTCF7-1 had a higher silencing efficiency than si-1ncTCF7-2; therefore, si-lncTCF7-1-transfected cells were used for subsequent experiments. Results of the CCK- 8 assay demonstrated that the proliferation of si-lncTCF7-transfected SW-620 and HT29 cells at 24, 48 and $72 \mathrm{~h}$ after transfection was significantly lower compared with si-NC-transfected SW-620 and HT29 cells at the same time points $(\mathrm{P}<0.05$; Fig. $2 \mathrm{~B})$. Cell cycle analysis demonstrated that lncTCF7 silencing induced a significant G1-phase arrest in SW-620 and HT29 cells ( $\mathrm{P}<0.05$; Fig. 2C and D). However, results of apoptosis analysis indicated that apoptosis was not significantly different between si-lncTCF7- and si-NC-transfected SW-620 and HT29 cells ( $>>0.05$; Fig. 2E and $\mathrm{F}$ ).

LncTCF7 silencing inhibits the migration and invasion of CRC cells. The migration and invasion of SW-620 and HT29 cells was determined by performing wound healing and Transwell assays, respectively, after $48 \mathrm{~h}$ transfection. The migration and invasion of cells was significantly lower among si-lncTCF7-transfected cells compared with si-NC-transfected cells ( $\mathrm{P}<0.05$; Fig. 3 ).

\section{Discussion}

The present study investigated the expression of lncTCF7 to assess its efficacy as a novel diagnostic marker of CRC. The results revealed that lncTCF7 expression was significantly higher in CRC tissues compared with adjacent normal tissues. In addition, lncTCF7 expression was significantly associated with tumor size, differentiation degree, TNM grade, lymph node metastasis and invasion depth; however, it was not significantly associated with sex, age, location and organization, indicating that lncTCF7 may be associated with tumorigenesis and tumor progression. These results are similar to those of previous studies, which reported that lncTCF7 was overexpressed in liver cancer and non-small cell lung cancer tissues $(9,16)$. Results of ROC analysis performed in the present study indicated that 1ncTCF7 may be an independent diagnostic marker of CRC.

In addition, the effect of lncTCF7 on CRC tumorigenesis and progression was investigated to determine its potential as a therapeutic target for CRC treatment. The results demonstrated that silencing of 1ncTCF7 inhibited the proliferation, 
migration and invasion, but did not significantly affect the apoptosis, of CRC cells. Additionally, cell cycle progression is an essential requirement for cell growth. In the current study, lncTCF7 silencing inhibited SW-620 and HT29 cell cycle transition from G1 to S phase, which indicated that lncTCF7 silencing may inhibit SW-620 and HT29 proliferation by suppressing cell cycle progression. Proliferation and cell cycle dysfunction serve an important role in CRC development, and cell migration and invasion serve a central role in CRC metastasis (22-24). The results of the present study also indicated that lncTCF7 silencing inhibited CRC development and metastasis, indicating that lncTCF7 may function as an oncogene to promote CRC development and metastasis. The results of the present study are consistent with those of previous studies. Wang et al (9) demonstrated that IncTCF7 promoted the self-renewal and propagation of liver cancer stem cells by recruiting the Switch/sucrose non-fermentable complex to the TCF7 promoter to upregulate TCF7 expression and to activate Wnt signaling. Furthermore, Wu and Wang (16) demonstrated that lncTCF7 increased the self-renewal and metastasis of human non-small cell lung cancer cells by promoting Slug and epithelial cell adhesion molecule expression. In another study, Wu et al (17) revealed that IncTCF7 increased the aggressiveness of hepatocellular carcinoma cells by activating epithelial-mesenchymal transition signaling.

However, there are several limitations to the data generated in the current study. Firstly, the overall survival of 1ncTCF7 on CRC prognosis was not analyzed due to the short follow-up time, and the overall survival of 1ncTCF7 on CRC prognosis may be investigated in further research. In addition, the effect of 1ncTCF7 on the animal models of CRC may be the focus of further research. Finally, further studies are required to determine the mechanism underlying lncTCF7-induced regulation of CRC tumorigenesis and progression. In conclusion, the results of the current indicated that lncTCF7 may be a potential biomarker and therapeutic target for CRC treatment.

\section{References}

1. Torre LA, Bray F, Siegel RL, Ferlay J, Lortet-Tieulent J and Jemal A: Global cancer statistics, 2012. CA Cancer J Clin 65: $87-108,2015$

2. Chen W, Zheng R, Zeng H, Zhang S and He J: Annual report on status of cancer in China, 2011. Chin J Cancer Res 27: $2-12,2015$

3. Fleshman JW and Smallwood N: Current concepts in rectal cancer. Clin Colon Rectal Surg 28: 5-11, 2015.

4. Rao AKDM, Rajkumar T and Mani S: Perspectives of long non-coding RNAs in cancer. Mol Biol Rep 44: 203-218, 2017.

5. Li M, Wang Y, Cheng L, Niu W, Zhao G, Raju JK, Huo J, Wu B, Yin B, Song Y and Bu R: Long non-coding RNAs in renal cell carcinoma: A systematic review and clinical implications. Oncotarget 8: 48424-48435, 2017.

6. Klingenberg M, Matsuda A,Diederichs S and Patel T: Non-coding RNA in hepatocellular carcinoma: Mechanisms, biomarkers and therapeutic targets. J Hepatol 67: 603-618, 2017.
7. Richtig G, Ehall B, Richtig E, Aigelsreiter A, Gutschner T and Pichler M: Function and clinical implications of long non-coding RNAs in melanoma. Int J Mol Sci 18: pii:E715, 2017.

8. Yang Y, Zhao L, Lei L, Lau WB, Lau B, Yang Q, Le X, Yang H, Wang C, Luo Z, et al: LncRNAs: The bridge linking RNA and colorectal cancer. Oncotarget 8: 12517-12532, 2017.

9. Wang Y, He L, Du Y, Zhu P, Huang G, Luo J, Yan X, Ye B, Li C, Xia P, et al: The long noncoding RNA lncTCF7 promotes self-renewal of human liver cancer stem cells through activation of Wnt signaling. Cell Stem Cell 16: 413-425, 2015.

10. Weber BN, Chi AW, Chavez A, Yashiro-Ohtani Y, Yang Q, Shestova O and Bhandoola A: A critical role for TCF-1 in T-lineage specification and differentiation. Nature 476: 63-68, 2011.

11. Cui L, Guan Y, Qu Z, Zhang J, Liao B, Ma B, Qian J, Li D, Li W, $\mathrm{Xu}$ GT and Jin Y: WNT signaling determines tumorigenicity and function of ESC-derived retinal progenitors. J Clin Invest 123: 1647-1661, 2013.

12. Nikuseva-Martic T, Serman L, Zeljko M, Vidas Z, Gašparov S, Zeljko HM, Kosović M and Pećina-Šlaus N: Expression of secreted frizzled-related protein 1 and 3, T-cell factor 1 and lymphoid enhancer factor 1 in clear cell renal cell carcinoma. Pathol Oncol Res 19: 545-551, 2013.

13. Siu MK, Chen WY, Tsai HY, Chen HY, Yin JJ, Chen CL, Tsai YC and Liu YN: TCF7 is suppressed by the androgen receptor via microRNA-1-mediated downregulation and is involved in the development of resistance to androgen deprivation in prostate cancer. Prostate Cancer Prostatic Dis 20: 172-178, 2017.

14. Chen WY, Liu SY, Chang YS, Yin JJ, Yeh HL, Mouhieddine TH, Hadadeh O, Abou-Kheir W and Liu YN: MicroRNA-34a regulates WNT/TCF7 signaling and inhibits bone metastasis in Ras-activated prostate cancer. Oncotarget 6: 441-457, 2015.

15. Wang Y, Zhang S, Xu Y, Zhang Y, Guan H, Li X, Li Y and Wang Y: Upregulation of miR-192 inhibits cell growth and invasion and induces cell apoptosis by targeting TCF7 in human osteosarcoma. Tumour Biol 37: 15211-15220, 2016.

16. Wu J and Wang D: Long noncoding RNA TCF7 promotes invasiveness and self-renewal of human non-small cell lung cancer cells. Hum Cell 30: 23-29, 2017.

17. Wu J, Zhang J, Shen B, Yin K, Xu J, Gao W and Zhang L: Long noncoding RNA lncTCF7, induced by IL-6/STAT3 transactivation, promotes hepatocellular carcinoma aggressiveness through epithelial-mesenchymal transition. J Exp Clin Cancer Res 34: $116,2015$.

18. Livak KJ and Schmittgen TD: Analysis of relative gene expression data using real-time quantitative PCR and the 2(-Delta Delta C(T)) method. Methods 25: 402-408, 2001.

19. Li C, Liang G, Yang S, Sui J, Yao W, Shen X, Zhang Y, Peng H, Hong W, Xu S, et al: Dysregulated lncRNA-UCA1 contributes to the progression of gastric cancer through regulation of the PI3K-Akt-mTOR signaling pathway. Oncotarget 8: 93476-93491, 2017.

20. Xu C, Sun L, Jiang C, Zhou H, Gu L, Liu Y and Xu Q: SPP1, analyzed by bioinformatics methods, promotes the metastasis in colorectal cancer by activating EMT pathway. Biomed Pharmacother 91: 1167-1177, 2017.

21. Pan Y, Jiao G, Wang C, Yang J and Yang W: MicroRNA-421 inhibits breast cancer metastasis by targeting metastasis associated 1. Biomed Pharmacother 83: 1398-1406, 2016.

22. Romagnoli P, Filipponi F, Bandettini L and Brugnola D: Increase of mitotic activity in the colonic mucosa of patients with colorectal cancer. Dis Colon Rectum 27: 305-308, 1984.

23. Paganelli GM, Biasco G, Santucci R, Brandi G, Lalli AA, Miglioli M and Barbara L: Rectal cell proliferation and colorectal cancer risk level in patients with nonfamilial adenomatous polyps of the large bowel. Cancer 68: 2451-2454, 1991.

24. Yao M, Lam EC, Kelly CR, Zhou W and Wolfe MM: Cyclooxygenase-2 selective inhibition with NS-398 suppresses proliferation and invasiveness and delays liver metastasis in colorectal cancer. Br J Cancer 90: 712-719, 2004. 